\title{
Radial Spin Helix in Two-Dimensional Electron Systems with Rashba Spin-Orbit Coupling
}

\author{
Yuriy V. Pershin* \\ Department of Physics and Astronomy and USC Nanocenter, \\ University of South Carolina, Columbia, SC 29208, USA \\ Valeriy A. Slipko \\ Department of Physics and Technology, V. N. Karazin Kharkov National University, Kharkov, Ukraine
}

\begin{abstract}
We suggest a long-lived spin polarization structure, a radial spin helix, and study its relaxation dynamics. For this purpose, starting with a simple and physically clear consideration of spin transport, we derive a system of equations for spin polarization density and find its general solution in the axially symmetric case. It is demonstrated that the radial spin helix of a certain period relaxes slower than homogeneous spin polarization and plain spin helix. Importantly, the spin polarization at the center of the radial spin helix stays almost unchanged at short times. At longer times, when the initial non-exponential relaxation region ends, the relaxation of the radial spin helix occurs with the same time constant as that describing the relaxation of the plain spin helix.
\end{abstract}

\section{INTRODUCTION}

At the present time, there is a significant interest in the field of electron spin relaxation in semiconductors stimulated by possible future applications of spins in electronics and computing $\stackrel{1,2}{\underline{2}}$. In many twodimensional (2D) electron systems the leading mechanism of spin relaxation is the D'yakonov-Perel' spin relaxation mechanism ${ }^{3,4}$. Within this mechanism, electron spins feel an effective momentum-dependent magnetic field randomized by electron scattering events resulting in relaxation of electron spin polarization. A number of theoretical and experimental studies on peculiarities of D'yakonov-Perel' spin relaxation were reported in the last decade $\mathrm{e}^{-16}$.

It was shown in Ref. 9 that the spin relaxation time for $2 \mathrm{D}$ electrons depends not only on material parameters (e.g., strength of spin-orbit interaction, electron mean free path, etc.) but also on the initial spin polarization profile. In particular, it was demonstrated that a plain spin helix in a $2 \mathrm{D}$ electron system with Rashba spinorbit interaction has a longer spin relaxation time than a homogeneous spin polarization ${ }^{9}$. A later study $\stackrel{11}{ }$ revealed that in a system with both Rashba 17 and Dresselhaus 18 interactions such an increase in spin relaxation time can be even more dramatic. This effect was also observed experimentally 13,19 .

In this paper, we consider spin relaxation of a radial spin helix in a 2D electron system with Rashba spin-orbit (SO) interaction. This structure is interesting because it provides, to the best of our knowledge, the longest spin relaxation time of $2 \mathrm{D}$ electrons subjected to Rashba SO interaction. Such a property is related to spin polarization in the vicinity of the special point of radial spin helix $r=0$. Physically, at short times, when the period of radial spin helix is equal to the period of spin precession, an electron diffusing from any direction to a point in the vicinity of $r=0$ has the same direction of spin polarization as the initial spin polarization at this point. Thus,
D'yakonov-Perel' spin relaxation becomes inefficient at short times for spin polarization in the vicinity of $r=0$.

In the radial spin helix, the initial distribution of spin polarization has a cylindrical (axial) symmetry and is given by

$$
\begin{array}{r}
S_{r}(r, t=0)=-S_{0} \sin (k r), \\
S_{\varphi}(r, t=0)=0, \\
S_{z}(r, t=0)=S_{0} \cos (k r),
\end{array}
$$

where $k$ is the wave vector, and $S_{0}$ is the initial amplitude of spin polarization. In Fig. 1(a) we show schematically spin polarization distribution in the radial direction. The overall distribution of $z$-component of the spin polariza-
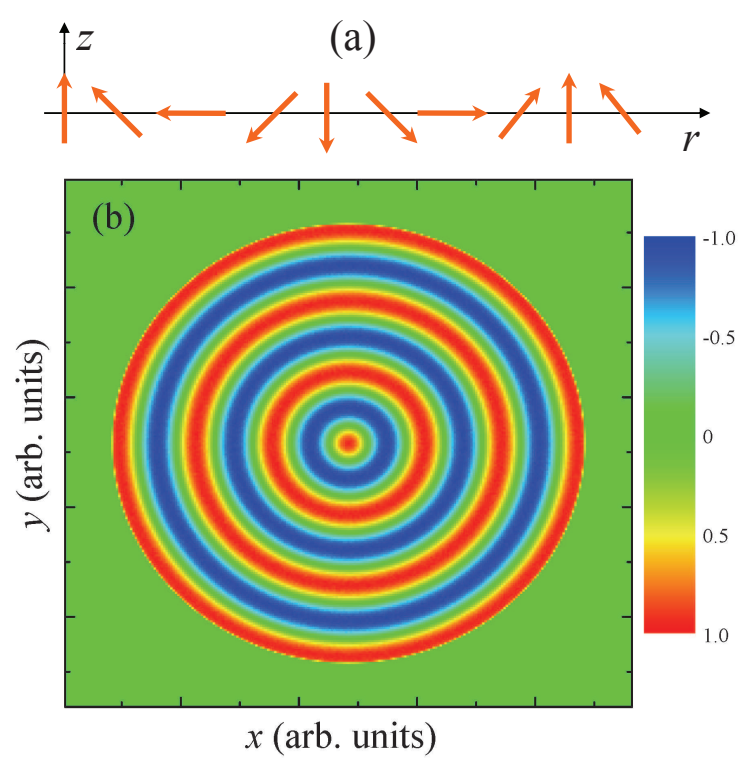

FIG. 1. (Color online) (a) Schematic of initial spin polarization distribution in a radial spin helix. (b) Initial distribution of $z$-component of spin polarization $\left(S_{z}(r, 0) / S_{0}\right)$ in the radial spin helix of finite radius used in our Monte Carlo simulations. 
tion in radial spin helix of finite radius is shown in Fig. 1(b). Moreover, in this paper we will often refer to a plain spin helix suggested in Ref. 9. The initial distribution of spin polarization components in the plain spin helix is

$$
\begin{array}{r}
S_{x}(x, t=0)=-S_{0} \sin (k x), \\
S_{y}(x, t=0)=0, \\
S_{z}(x, t=0)=S_{0} \cos (k x) .
\end{array}
$$

This paper is organized as follows. In Sec. II, a driftdiffusion equation approach is used to study relaxation dynamics of the radial spin helix. We obtain general expressions for spin polarization as a function of time and study its short and long time behavior. Our analytical studies are supported by Monte Carlo simulations presented in Sec. III Our main results and conclusions are summarized in Sec. IV] Moreover, in several Appendices following the main text, we provide additional calculation details. Specifically, electron spin rotations induced by Rashba SO interaction are considered in the Appendix A, spin drift-diffusion equations are derived in the Appendix B a general analytical solution of spin drift-diffusion equation in the axially symmetric case is presented in the Appendix $\mathrm{C}$, and relaxation of plain spin helix is discussed in the Appendix D.

\section{DRIFT-DIFFUSION DESCRIPTION OF RADIAL SPIN HELIX}

In this section we consider the relaxation dynamics of the radial spin helix analytically. The initial spin polarization in the radial spin helix is of cylindrical symmetry and described by Eqs. (1] 33). Intuitively, a special point in the radial spin helix is $r=0$, because the electrons motion through this point along straight trajectories in all directions should not lead to spin relaxation at short times for a specific value of the wave vector $k$. Correspondingly, the spin lifetime of electrons located in a region within $r=0$ should be longer than the spin lifetime of homogeneous spin polarization and of plain spin helix. This effect is in the focus of our investigation.

Let us consider a two-dimensional electrons confined in a quantum well or heterostructure with Rashba-type spin-orbit interaction $\underline{17}$. The standard Hamiltonian with the Rashba term is given by

$$
\hat{H}=\frac{\hat{\mathbf{p}}^{2}}{2 m}+\alpha(\hat{\sigma} \times \hat{\mathbf{p}}) \cdot \mathbf{z},
$$

where $\hat{\mathbf{p}}=\left(\hat{p}_{x}, \hat{p}_{y}\right)$ is the $2 \mathrm{D}$ electron momentum operator, $m$ is the effective electron's mass, $\hat{\sigma}$ is the Paulimatrix vector, $\alpha$ is the spin-orbit coupling constant and $\mathbf{z}$ is a unit vector perpendicular to the confinement plane.

It is not difficult to show that in the case of Hamiltonian (7) the quantum mechanical evolution of a spin of an electron with a momentum $\mathbf{p}$ can be reduced to a spin rotation with the angular velocity $\Omega=2 \alpha p / \hbar$ about the axis determined by the unit vector $\mathbf{n}=\mathbf{p} \times \mathbf{z} / p$ (see Appendix (A). In this way, the spin-orbit coupling constant $\alpha$ enters into equations through the parameter $\eta=2 \alpha m \hbar^{-1}$, which gives the spin precession angle per unit length.

Besides this evolution, 2D electrons experience different bulk scattering events such as, for example, due to phonons or impurities. These scatterings randomize the electron trajectories. Correspondingly, the direction of spin rotation becomes fluctuating what causes average spin relaxation (dephasing). This is the famous D'yakonov-Perel' spin relaxation mechanism ?.4 $^{3.4}$ The time scale of the bulk scattering events can then be characterized by a single rate parameter, the momentum relaxation time $\tau$. It is connected to the mean free path by $\ell=v \tau$, where $v=p / m$ is the mean electron velocity. To take into account these scatterings we use a model of diffusive spin transport, which in the limit of small $k \ell \ll 1$, yields the spin drift-diffusion equations (B3) B55 (Appendix B provides derivation details).

Let us consider dynamics of a radial spin helix relaxation. We assume that such a structure is created at the initial moment of time with the spin polarization components given by Eqs. (1/3). The exact solution of the radial spin drift-diffusion equations (C1) C2) with initial conditions (113) and constants $\gamma$ and $C$ from Eqs. (B6) can be written as (see Appendix $\mathbb{C}$ for more details)

$$
\begin{aligned}
& \frac{S_{r}(r, t)}{S_{0}}=-\frac{d}{d k} \int_{0}^{k} \frac{d s J_{1}(s r)}{\sqrt{k^{2}-s^{2}}}\left[k \cosh \left(\sqrt{\eta^{2}+16 s^{2}} \frac{\eta D t}{2}\right)\right. \\
& \left.+\left(k \eta+4 s^{2}\right) \frac{\sinh \left(\sqrt{\eta^{2}+16 s^{2}} \frac{\eta D t}{2}\right)}{\sqrt{\eta^{2}+16 s^{2}}}\right] e^{-\left(s^{2}+3 \eta^{2} / 2\right) D t},(8) \\
& \quad \frac{S_{z}(r, t)}{S_{0}}=\frac{d}{d k} \int_{0}^{k} \frac{d s s J_{0}(s r)}{\sqrt{k^{2}-s^{2}}}\left[\cosh \left(\sqrt{\eta^{2}+16 s^{2}} \frac{\eta D t}{2}\right)\right. \\
& \left.+(4 k-\eta) \frac{\sinh \left(\sqrt{\eta^{2}+16 s^{2}} \frac{\eta D t}{2}\right)}{\sqrt{\eta^{2}+16 s^{2}}}\right] e^{-\left(s^{2}+3 \eta^{2} / 2\right) D t},
\end{aligned}
$$

where $J_{1}(r)$ and $J_{0}(r)$ are the Bessel functions of the first and zeroth order correspondingly and $D$ is the diffusion constant.

Eqs. (8-9) define completely the radial spin helix at any point $r$ and at any moment of time $t$. The time dependence of spin polarization at the center of helix is of particular interest because the spin relaxation at this point is the slowest. The radial component of spin polarization $S_{r}$ in the vicinity of $r=0$ is close to zero (it follows from symmetry considerations or directly from Eq. (81)). Therefore, below, we derive asymptotic expressions at short and long times for $S_{z}$ only. At short times $D \eta^{2} t \ll 1$, an expansion of the RHS of Eq. (9) in 
$t$ and its integration over $s$ at $r=0$ results in

$$
\begin{aligned}
\frac{S_{z}(0, t)}{S_{0}}= & 1-2(k-\eta)^{2} D t+\frac{2}{3}(k-\eta)\left(2 k^{3}-6 k^{2} \eta\right. \\
+6 k \eta^{2}- & \left.3 \eta^{3}\right) D^{2} t^{2}-\left(\frac{8}{15} k^{6}-\frac{16}{5} k^{5} \eta+8 k^{4} \eta^{2}-\frac{104}{9} k^{3} \eta^{3}\right. \\
& \left.+\frac{32}{3} k^{2} \eta^{4}-\frac{14}{3} k \eta^{5}+\frac{4}{3} \eta^{6}\right) D^{3} t^{3}+O\left(t^{4}\right)
\end{aligned}
$$

It follows from Eq. (10) that when the radial spin helix period is equal to spin precession length (this happens when $k=\eta$ ) the decay of $S_{z}$ at the center of helix starts with a cubic term in $t$

$$
\frac{S_{z}(0, t)}{S_{0}}=1-\frac{10}{9}\left(D \eta^{2} t\right)^{3}+O\left(t^{4}\right), \text { for } k=\eta, D \eta^{2} t \ll 1 \text {. }
$$

This means that the spin relaxation at the center of the radial spin helix at short times is significantly suppressed (for this special wave number, $k=\eta$ ) and characterized by a rather long initial interval of non-exponential behaviour.

The asymptotic behaviour of $S_{r}$ and $S_{z}$ at long times, $D \eta^{2} t \gg 1$, can be determined by taking into account the dominant contribution to the integrals in Eqs. (8) and (9). This contribution comes from the vicinity of a point $s \in[0, k]$ corresponding to the maximum of the $-\lambda_{-}(s)$ in the integration interval (see Eq. (C10) and Fig. 7). We should consider three cases. In the first case, when $0<$ $k<k_{m}=\sqrt{15} \eta / 4$, the main contribution to the integrals in Eqs. (8) and (9) comes from the vicinity of point $s=k$ at the right end of the integration interval. In the second case, when $k=k_{m}$, we should keep in mind that $k=$ $k_{m}$ is a stationary point of $\lambda_{-}(s)$ entering the exponent. Therefore, in this case, the asymptotic behaviour differs from the case $k<k_{m}$ by a pre-exponential factor. In the third case, when $k>k_{m}$, the main contribution to the integrals in Eqs. (8) and (9) arises from the vicinity of the inner stationary point $s=k_{m}$ of $\lambda_{-}(s)$.

The asymptotic behaviour of Laplace-type integrals is obtained using standard for this purpose technics. From Eq. (9) we get

$$
\begin{aligned}
& \frac{S_{z}(r, t)}{S_{0}}=\left(1+\frac{4 k-\eta}{\sqrt{\eta^{2}+16 k^{2}}}\right) J_{0}(k r) \sqrt{-\frac{\pi k}{8} \frac{d \lambda_{-}(k)}{d k} t} \\
& \times e^{-\lambda_{-}(k) t}, \text { for } 0<k<\frac{\sqrt{15}}{4} \eta,-k \frac{d \lambda_{-}(k)}{d k} t \gg 1,(12) \\
& \quad \frac{S_{z}(r, t)}{S_{0}}=\frac{\sqrt{15}(\sqrt{15}+3)}{32 \sqrt{2}} \Gamma\left(\frac{3}{4}\right) J_{0}\left(k_{m} r\right)\left(D \eta^{2} t\right)^{\frac{1}{4}} \\
& \times e^{-\lambda_{-}\left(k_{m}\right) t}, \text { for } k=k_{m}=\frac{\sqrt{15}}{4} \eta, D \eta^{2} t \gg 1,(13) \\
& \frac{S_{z}(r, t)}{S_{0}}=-\frac{3}{32} \frac{(4 k+5 \eta) \eta}{\left(k^{2}-k_{m}^{2}\right)^{3 / 2}} J_{0}\left(k_{m} r\right) \sqrt{\frac{\pi}{D t}} e^{-\lambda-\left(k_{m}\right) t} \\
& \text { for } k>\frac{\sqrt{15}}{4} \eta,\left(k-k_{m}\right)^{2} D t \gg 1, D \eta^{2} t \gg 1 . \quad(14)
\end{aligned}
$$

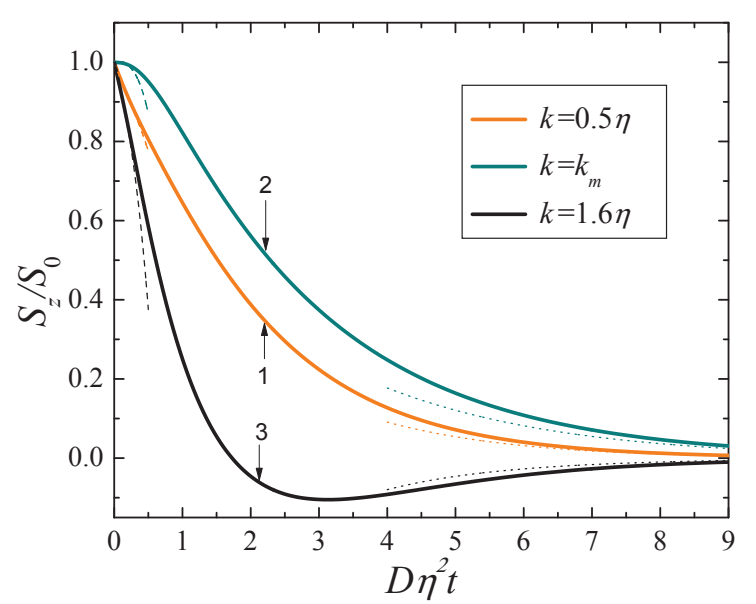

FIG. 2. (Color online) Time-dependence of $S_{z}(0, t)$ in the radial spin helix at several values of $k$.

From Eq. (12) we see that if the wave vector $k$ satisfies the inequality $0<k<k_{m}$, then, at long times, the spin polarization decay is mainly exponential and characterized by a relaxation time $\tau(k)=\left(\lambda_{-}(k)\right)^{-1}$. This relaxation time increases with $k$. At the same time (accordingly to Eq. (10)), the spin polarization decay at short times also decreases with $k$. Therefore, the spin life time of the radial spin helix increases with increase of $k \in\left(0, k_{m}\right)$.

At $k=k_{m}$, as it follows from Eqs. (13]14), the relaxation time reaches its maximum value $\tau_{m}=$ $\left(\lambda_{-}\left(k_{m}\right)\right)^{-1}=\left(7 D \eta^{2} / 16\right)^{-1}$. Moreover, since $k_{m} \approx$ $0.97 \eta$ is very close to $\eta$, the conditions for short time suppression of spin relaxation are almost optimal at this value of $k$. Therefore, when $k=k_{m}$, the spin relaxation is significantly suppressed at both short and long times.

When $k>k_{m}$, the asymptotic relaxation time is the same as when $k=k_{m}$ (see Eqs. (1314)). However, even when $k$ is close to $k_{m}$, the ratio of the absolute value of RHS of Eq. (14) to those of Eq. (13) is small (of the order of $\left.\left[\left(k-k_{m}\right)^{2} D t\right]^{-3 / 4}\right)$. In addition, in the asymptotic formula (14), the pre-exponential factor changes its sign. Therefore, the spin polarization $S_{z}$ must turn to zero at some moment of time, before it reaches the asymptotic behaviour given by Eq. (14). Eq. (10) at $k>\eta \approx k_{m}$ also predicts a relaxation increase with $k$ when $k>k_{m}$.

Thus we conclude that the longest spin relaxation time for the spin polarization at the center of the radial spin helix occurrs at the wave vector

$$
k=k_{m}=\sqrt{15} \eta / 4
$$

and is given by

$$
\tau_{m}=\left(7 D \eta^{2} / 16\right)^{-1}
$$

In addition, at this value of $k$, the dynamics of spin polarization in the vicinity of $r=0$ is non-exponential at short times, when the spin polarization remains almost constant. These are the main results of our calculations. 
(a)

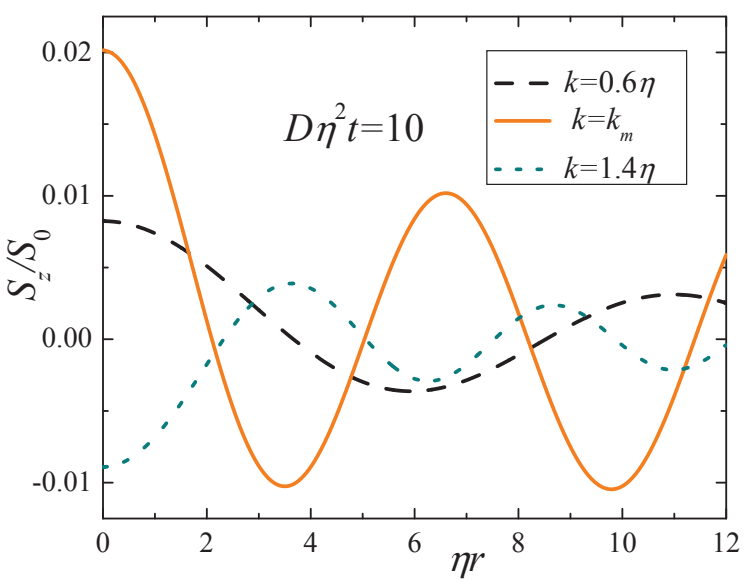

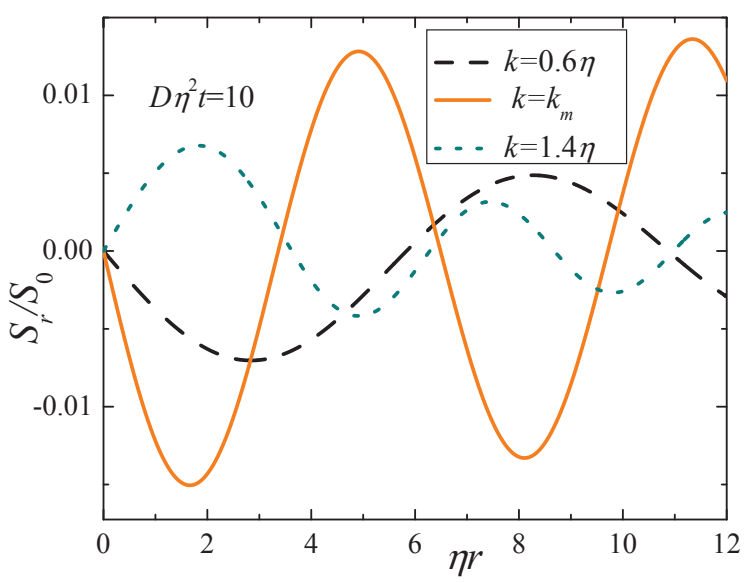

FIG. 3. Spatial dependence of $S_{z}$ (a) and $S_{r}$ (b) in the radial spin helix at the indicated moment of time.

The relaxation of initially homogeneous spin polarization (when $k=0$ ) can be obtained from Eq. (9) in the limit $k \rightarrow 0$. In this limiting case the factor before the exponential function $e^{-\lambda_{-}(s) t}$ in Eq. (9) turns to zero in $k=0$ limit. Therefore, at $k=0$, the spin relaxation is determined by the exponential function $e^{-\lambda_{+}(0) t}$, where, accordingly to Eq. (C10), $\lambda_{+}(0)=2 D \eta^{2}$.

In fact, the exact time dependence of $S_{z}$ at $k=0$ coincides with its asymptotic behaviour. It can be clearly seen from both Eqs. (9) and (B5) that

$$
S_{z}(t)=S_{0} e^{-\lambda_{+}(0) t}=S_{0} e^{-2 D \eta^{2} t}, \text { for } k=0 .
$$

Accordingly to Eq. (17), the initially homogeneous spin polarization, directed along $z$-axis, decays exponentially with a time constant $\tau^{(h)}=\left(2 D \eta^{2}\right)^{-1}$. We also note that the applicability limits of the long times asymptotic expressions listed in Eqs. (12]14) do not allow calculations of $S_{z}$ at $k=0$ or $k=k_{m}$ as a limiting case of Eq. (12).

In Fig. 2 we show the time dependence of the spin polarization component $S_{z}(r=0, t)$ calculated at several wave vectors $(k=0.5 \eta$ (solid line 1$), k=k_{m}=\sqrt{15} \eta / 4$ (solid line 2) and $k=1.6 \eta$ (solid line 3)) using Eq. (9). The dashed lines 1, 2 and 3 represent asymptotic behavior at short times (given by Eq. (10)), and dotted lines $1,2,3$ show asymptotic behavior at long times (given by Eqs. (12,14) ) for the same values of the wave vectors $k$. These curves reveal main features discussed above.

The spatial dependences of $z$ - and $r$-components of spin polarization, calculated from Eqs. (9) and (8), are presented in Fig. 3 for a particular moment of time $D \eta^{2} t=10$ and wave vectors $k=0.6 \eta$ (dashed lines), $k=k_{m}$ (solid lines), and $k=1.4 \eta$ (dotted lines). These plots clearly demonstrate that the maxima of $S_{z}$ and $S_{r}$ are reached at the wave vector $k=k_{m}$.

Fig. 4 depicts time dependence of $S_{z}(r=0, t)$ for homogeneous spin polarization (calculated from Eq. (17)), plain spin helix (calculated from Eq. (D4) ) and radial

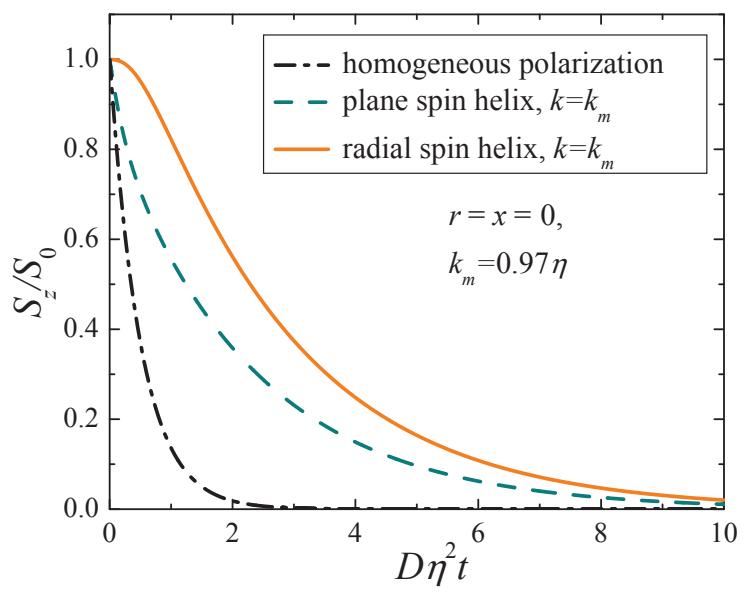

FIG. 4. (Color online) Time dependence of $S_{z}(r=0, t)$ for three different initial spin configurations: homogeneous polarization, plain spin helix and radial spin helix. The radial spin helix is characterized by the largest magnitude of spin polarization at any moment of time.

spin helix. This plot demonstrates that the spin polarization at $r=0$ in the radial spin helix lives longer that those in the case of homogeneous spin polarization and plain spin helix. It is interesting and important that at short times this curve stays almost flat as expected. At longer times, when the initial non-exponential relaxation region ends, the relaxation of spin polarization in the radial spin helix occurs with the same time constant as those of the plain spin helix (it can be shown analytically from Eqs. (D3 D4 that the longest spin relaxation time of the plain spin helix is given by Eq. (16) and occurs at $k=k_{m}$ given by Eq. (15)). Therefore, in both cases, the increase of the exponential relaxation time relative to the homogeneous spin polarization is equal to $32 / 7$. 

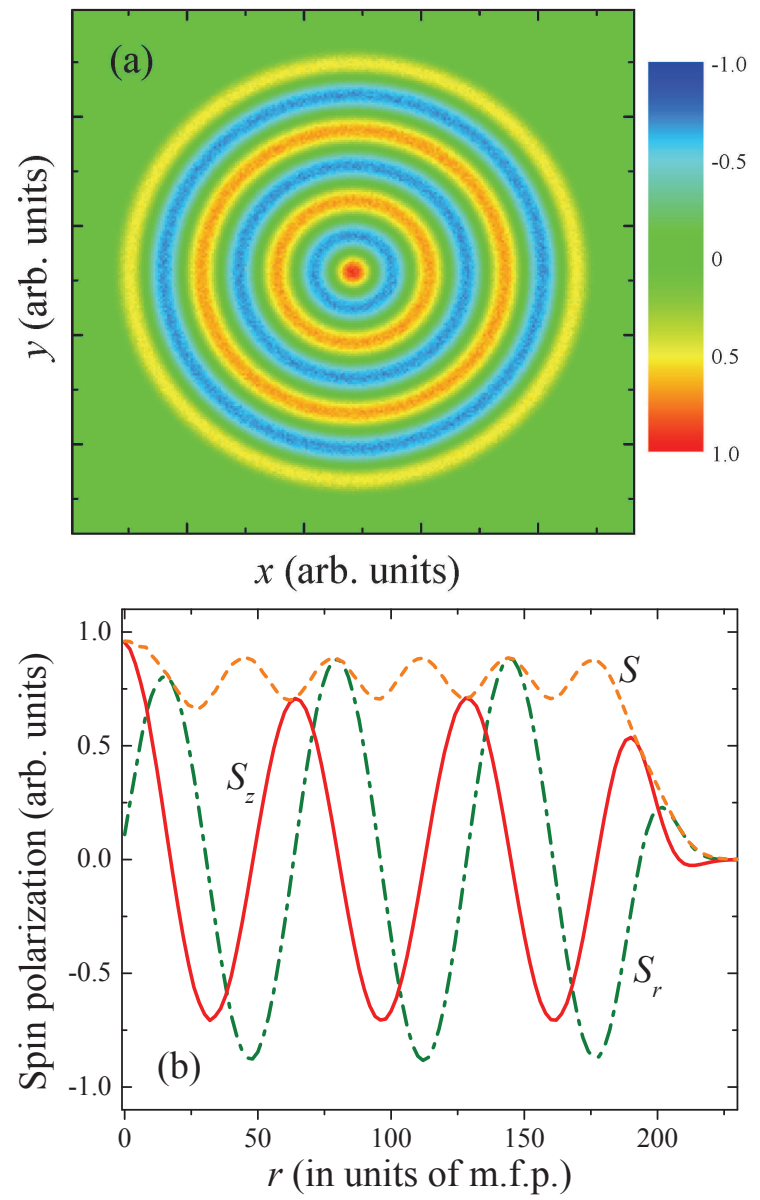

FIG. 5. (Color online) Distributions of $z$ component (a) and all components (b) of spin polarization $\left(S_{\varphi}=0, S=\right.$ $\left.\left(S_{r}^{2}+S_{z}^{2}\right)^{1 / 2}\right)$ at $t=100 \tau$ in the radial spin helix. The red (dark) area in the center of radial spin helix in (a) and the maximum of $S$ and $S_{z}$ in the vicinity of $r=0$ in (b) demonstrate a longer spin lifetime of electrons located in this region. This plot was obtained at $\eta \ell=0.1$ and the radial spin helix period $a=64.77 \ell$ (this value of $a$ corresponds to $k=k_{m}$ ). M.f.p. (mean free path) stands for $\ell$.

\section{MONTE CARLO SIMULATIONS}

In order to obtain an additional insight on spin relaxation of the radial spin helix, we perform Monte Carlo simulations employing an approach described in Refs. $[5$ and 20. This Monte Carlo simulation method uses a semiclassical description of electron space motion and quantum-mechanical description of spin dynamics (the later is based on the Hamiltonian (7)). All specific details of the Monte Carlo simulations program can be found in the references cited above and will not be repeated here. To some extent, Monte Carlo simulations program numerically solves Eqs. (B3) B55) taking into account relations (A4) and (A5).

All numerical results related to the radial spin helix were obtained using an ensemble of $10^{8}$ electrons initially

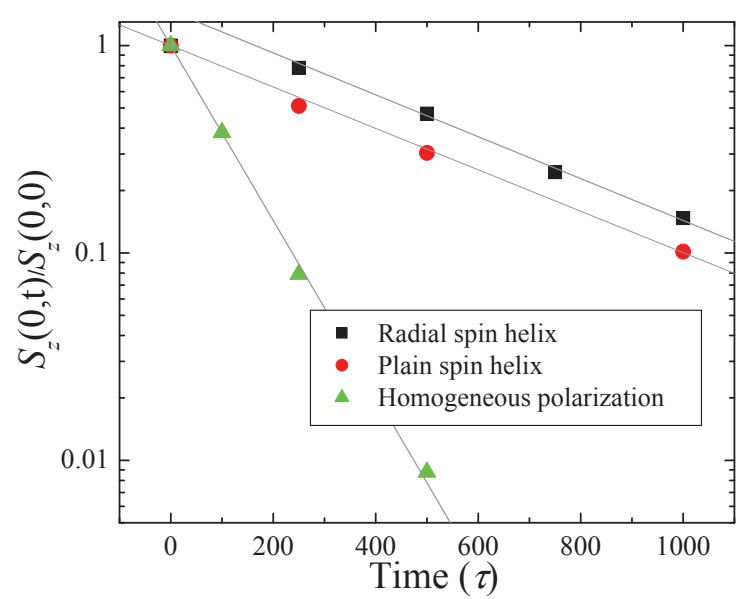

FIG. 6. (Color online) Spin polarization as a function of time for different initial spin polarization configurations. This plot was obtained using the parameters values $\eta \ell=0.1$ and $a=$ $64.77 \ell$ (the period of helices). The straight lines are fitting curves selected as $\exp \left(-\left(t-t_{0}\right) / t_{1}\right)$. The parameters of the fitting curves are $t_{0}=0, t_{1}=103 \tau$ for homogeneous spin polarization, $t_{0}=0 \tau, t_{1}=435 \tau$ for plain spin helix, and $t_{0}=165 \tau, t_{1}=430 \tau$ for radial spin helix.

homogeneously distributed within a circle of a sufficiently large radius $R=200 \ell$ to insure that the influence of boundary effects on spin polarization in a region in the vicinity of $r=0$ is negligible. The initial configuration of $z$-component of spin polarization of these electrons is presented in Fig. 1(b). Our Monte Carlo simulations results are in an excellent agreement with the theory of radial spin helix relaxation presented above.

Fig. 5)(a) demonstrates the spatial dependence of $S_{z}$ in the radial spin helix at $t=100 \tau$ and Fig. [5(b) depicts the radial dependence of the spin polarization components at the same moment of time. In particular, it can be clearly seen that the decay of $S_{z}$ in the vicinity of $r=0$ is slower than in other regions. The total spin polarization $S$ shows oscillations that are related to a well-known feature of D'yakonov-Perel' relaxation: the spin relaxation time of the perpendicular to plane spin polarization is shorter than the spin relaxation time of the in-plane spin polarization. Similar oscillation of spin polarization amplitude were previously found in the relaxation dynamics of the plain spin helix. ${ }^{\frac{9}{}}$

In addition to the radial spin helix relaxation, we simulated the relaxation of homogeneous spin polarization and relaxation of plain spin helix. Monte Carlo simulations reveal that at long times the time dependence of spin polarization in all spin configurations (homogeneous spin polarization, plain spin helix and radial spin helix) exhibits an exponential decay.

In Fig. 6 we compare the time dependencies of spin polarization $\left(S(0, t) / S_{0}\right)$ for three different initial polarization configurations: the homogeneous spin polarization (initial spin polarization is selected as $S_{x}=S_{y}=0$, $S_{z}=S_{0}$ ), plain spin helix and radial spin helix. The 
selected period of radial and plane spin helices $\left(k=k_{m}\right)$ corresponds to the longest spin lifetime of these structures at a fixed $\eta \ell=0.1$. It follows from Fig. 6 that the spin polarization in the radial spin helix is the most robust against the relaxation. We emphasize that the long-time behavior of all curves can be perfectly fitted by an exponential law given in the caption of Fig. 6, The numerically obtained increase in the spin lifetime of the radial spin helix $435 / 103 \sim 4.2$ is very close to the theoretically predicted value $32 / 7 \sim 4.6$. We also note that a slightly longer spin relaxation time increase $(\sim 6)$ reported in Ref. 9 for the plain spin helix can be related to a large value of $\eta \ell=0.3$ that is beyond the linear spin drift-diffusion theory.

\section{CONCLUSION}

The dynamics of spin relaxation of radial spin helix was investigated using spin drift-diffusion equations and Monte Carlo simulations. Starting with a clear model of diffusive spin transport, we derived spin drift-diffusion equations for electron spin polarization in $2 \mathrm{D}$ semiconductor structures with Rashba spin-orbit coupling. The general solution of these equations for the axial symmetric case was found. Based on this solution, we studied the evolution of the suggested long-lived spin structure the radial spin helix. It was shown that the relaxation of spin polarization in the vicinity of $r=0$ in this structure demonstrates an unusual long initial non-exponential relaxation behavior followed by an exponential decay. The optimal value of the radial spin helix wave vector was found and corresponding exponential relaxation time was calculated. Qualitatively, the initial non-exponential decay feature can be explained by existence of an infinite set of dephasing-free trajectories propagating through the point $r=0$.

In order to additionally check our analytical results, we also performed Monte Carlo simulations of the dynamics of radial spin helix relaxation using the same Monte Carlo simulation technique as those described in Refs. 5 and 20. Fig. [5]shows a representative result of our simulation in which it is clearly demonstrated that the polarization decay at $r=0$ is slowest. Our analytical and Monte Carlo simulations results are in a perfect agreement.

To conclude, the radial spin helix is a new structure exhibiting an unusual spin relaxation dynamics and relatively long lifetime. Its property of slow relaxation dynamics at short times is very interesting. Experimentally, the radial spin helix can be created by spin injection from a point electrode located at the center of a second ringshape electrode or possibly by a modified spin gratings technique ${ }^{21}$.
Appendix A: Rotation of a single spin subjected to Rashba SO interaction

Let us consider an electron with a momentum $\mathbf{p}=$ $\left(p_{x}, p_{y}\right)$. The Hamiltonian (7) can be rewritten as

$$
\hat{H}=\frac{\mathbf{p}^{2}}{2 m}+\alpha p \mathbf{n} \cdot \hat{\sigma},
$$

where $\mathbf{n}=\mathbf{p} \times \mathbf{z} / p$ is the unit vector.

Since eigenvalues spin projection operator $\hat{\mathbf{s}} \cdot \mathbf{n}=\hat{\sigma} \cdot \mathbf{n} / 2$ on any direction are equal to $\pm 1 / 2$, the energy levels of (A1) are given by

$$
E_{ \pm}=\frac{p^{2}}{2 m} \pm \alpha p
$$

and it is clear that corresponding spin eigenfunctions are the states with spin directed along and opposite to $\mathbf{n}$.

The evolution operator acting only on spin variables of the electron with momentum $\mathbf{p}$ is equal to

$$
\hat{U}(t)=e^{-i \hat{H} t / \hbar}=\exp \left\{\frac{-i \mathbf{p}^{2} t}{2 m \hbar}\right\} \exp \left\{-i \Omega t \mathbf{n} \cdot \frac{\hat{\sigma}}{2}\right\},
$$

where $\Omega=2 \alpha p / \hbar$.

We note that the evolution operator $\mathrm{A3}$ coincides with the operator of finite rotation by an angle $\Omega t=$ $2 \alpha p t / \hbar$ about $\mathbf{n}$-axis up to the phase factor $e^{-i p^{2} t /(2 m \hbar)}$. Since electron spin $s$ transforms under rotations as the ordinary vector, we obtain

$\mathbf{s}(t)=\cos (\Omega t) \mathbf{s}(0)+\sin (\Omega t) \mathbf{n} \times \mathbf{s}(0)+2 \sin ^{2}(\Omega t / 2) \mathbf{n} \cdot \mathbf{s}(0) \mathbf{n}$,

where

$$
\Omega=2 \alpha p / \hbar, \quad \mathbf{n}=\mathbf{p} \times \mathbf{z} / p .
$$

\section{Appendix B: Derivation of spin drift-diffusion equations}

Let us consider a two-dimensional non-degenerate electron gas and use a semiclassical approach to model the electron space motion and quantum-mechanical approach based on the Hamiltonian (77) to describe the electron spin dynamics. Moreover, we assume the electrical neutrality and absence of an external electromagnetic field. Within our approach, 2D electrons are characterized by the momentum relaxation time $\tau$ and the mean free path $\ell$, so that the average velocity of electrons is $v=\ell / \tau$. From elementary gas-kinetic considerations ${ }^{22}$ we can write an equation for the change of electron spin polarization $\Delta \mathbf{S}(x, y, t)$ in a region of dimensions $2 \ell \times 2 \ell$ with the center at $(x, y)$ during the time interval $\tau$ :

$$
\begin{array}{r}
(2 \ell)^{2} \Delta \mathbf{S}(x, y, t)=\frac{1}{4} v \tau(2 \ell)\left\{\mathbf{S}^{\prime}(x-2 \ell, y, t)\right. \\
+\mathbf{S}^{\prime}(x+2 \ell, y, t)+\mathbf{S}^{\prime}(x, y-2 \ell, t)+\mathbf{S}^{\prime}(x, y+2 \ell, t)(\mathrm{B} 1) \\
-4 \mathbf{S}(x, y, t)\} .
\end{array}
$$


In the right hand side of Eq. (B1), the first four terms are the spin polarization fluxes into the region from four sides with length $2 \ell$, and the last term is the flux out of this region. The prime symbols in Eq. (B1) denote a change of spin polarization because of SO interactioninduced spin precession by the angle $2 \Omega \tau=4 \alpha \mathrm{m} \ell / \hbar=$ $2 \eta \ell$ accordingly to Eqs. A4/A5 . For example,

$$
\begin{aligned}
& \mathbf{S}^{\prime}(x-2 \ell, y, t)=\cos (2 \eta \ell) \mathbf{S}(x-2 \ell, y, t)-\sin (2 \eta \ell) \mathbf{y} \\
& \quad \times \mathbf{S}(x-2 \ell, y, t)+2 \sin ^{2}(\eta \ell) \mathbf{y} \cdot \mathbf{S}(x-2 \ell, y, t) \mathbf{y},(\mathrm{B} 2)
\end{aligned}
$$

where $\mathbf{y}$ is the unit vector along $y$-axis.

In order to obtain drift-diffusion equations for spin polarization, we substitute expressions for $\mathbf{S}^{\prime}$ into Eq. (B1), and expand trigonometrical functions up to quadric terms with respect to small $2 \eta \ell$ and $\mathbf{S}^{\prime}$ terms up to quadric terms with respect to $2 \ell$. The resulting system of drift-diffusion equations for spin polarization have a form

$$
\begin{array}{r}
\frac{\partial S_{x}}{\partial t}=D \Delta S_{x}+C \frac{\partial S_{z}}{\partial x}-2 \gamma S_{x} \\
\frac{\partial S_{y}}{\partial t}=D \Delta S_{y}+C \frac{\partial S_{z}}{\partial y}-2 \gamma S_{y} \\
\frac{\partial S_{z}}{\partial t}=D \Delta S_{z}-C\left(\frac{\partial S_{x}}{\partial x}+\frac{\partial S_{y}}{\partial y}\right)-4 \gamma S_{z}
\end{array}
$$

where

$$
C=2 \eta D, \quad \gamma=\frac{1}{2} \eta^{2} D
$$

and

$$
D=\frac{\ell^{2}}{2 \tau} .
$$

Here $D$ is the coefficient of diffusion, $C$ describes spin rotations, and $\gamma$ is the coefficient describing spin relaxation.

It is interesting to note that the same drift-diffusion equations (B3, B6 can be obtained for the model of $2 \mathrm{D}$ localized electrons on a lattice ${ }^{23}$ in the hopping regime. However, in this case, the diffusion coefficient is equal to $D=\ell^{2} /(4 \tau)$, where $\tau$ is the characteristic hopping time and $\ell$ is the distance between lattice sites.

\section{Appendix C: Analytical solution of drift-diffusion equations in the axially symmetric case}

In the axially symmetric case (assuming that $S_{r}=$ $S_{r}(r, t), S_{z}=S_{z}(r, t)$ and $S_{\varphi}=0$ ) Eqs. (B3, B5 can be written as

$$
\begin{gathered}
\frac{\partial S_{r}}{\partial t}=D\left[\frac{\partial}{r \partial r}\left(r \frac{\partial S_{r}}{\partial r}\right)-\frac{S_{r}}{r^{2}}\right]+C \frac{\partial S_{z}}{\partial r}-2 \gamma S_{r} \\
\frac{\partial S_{z}}{\partial t}=D \frac{\partial}{r \partial r}\left(r \frac{\partial S_{z}}{\partial r}\right)-C \frac{\partial\left(r S_{r}\right)}{r \partial r}-4 \gamma S_{z}
\end{gathered}
$$

Let us find a general solution of Eqs. C1 C2 for the case of an infinite plane. We search a specific solution of the above Eqs. in the form

$$
\begin{aligned}
& S_{r}(r, t)=A(s, t) J_{1}(s r), \\
& S_{z}(r, t)=B(s, t) J_{0}(s r),
\end{aligned}
$$

where $J_{1}(r)$ and $J_{0}(r)$ are the Bessel functions of the first and zeroth order correspondingly. Substituting expressions (C3 C4 into Eqs. (C1,C2) we obtain a system of ordinary differential equations for unknown functions $A(s, t)$ and $B(s, t)$ of positive parameter $s$ and time $t$

$$
\begin{aligned}
& \frac{d A(s, t)}{d t}=-\left(D s^{2}+2 \gamma\right) A(s, t)-C s B(s, t), \\
& \frac{d B(s, t)}{d t}=-C s A(s, t)-\left(D s^{2}+4 \gamma\right) B(s, t) .
\end{aligned}
$$

The general solution of this system can be presented as

$$
\begin{array}{r}
A(s, t)=C_{+}(s) s C e^{-\lambda_{+}(s) t}+C_{-}(s) s C e^{-\lambda_{-}(s) t}, \\
B(s, t)=C_{+}(s)\left(\gamma+\sqrt{\gamma^{2}+C^{2} s^{2}}\right) e^{-\lambda_{+}(s) t} \\
+C_{-}(s)\left(\gamma-\sqrt{\gamma^{2}+C^{2} s^{2}}\right) e^{-\lambda_{-}(s) t},
\end{array}
$$

where we denote

$$
\lambda_{ \pm}(s)=D s^{2}+3 \gamma \pm \sqrt{\gamma^{2}+C^{2} s^{2}},
$$

and $C_{ \pm}(s)$ are arbitrary functions of positive parameter $s$. Using Eqs. (B6), Eq. (C9) can be rewritten in a more simple form

$$
\lambda_{ \pm}(s)=\frac{1}{2} D\left(2 s^{2}+3 \eta^{2} \pm \eta \sqrt{\eta^{2}+16 s^{2}}\right) .
$$

The special solutions (C3) (with $A(s, t)$ and $B(s, t)$ given by Eqs. (C7,C9) of the radial drift-diffusion equations C1 C2 have the following simple meaning. Accordingly to Eqs. (C3 C4 the spatial dependencies of the radial and $z$-components of spin polarization are proportional to the first and zeroth order Bessel function at any moment of time. The parameter $s$ is similar to the wave vector $k$ for the plane case. The amplitudes $A(s, t)$ and $B(s, t)$ determine the time dependence of the radial and $z$-components of spin polarization. If $C_{+}(s) \neq 0, C_{-}(s)=0\left(C_{+}(s)=0, C_{-}(s) \neq 0\right)$, then these amplitudes are exponential functions of time with the inverse relaxation time $\lambda_{+}(s)\left(\lambda_{-}(s)\right)$ as it can be seen from Eqs. (C7,C8).

Whereas the inverse relaxation time $\lambda_{+}(s)$ takes its minimum value at $s=0$ and monotonically increases with parameter $s$, the inverse relaxation time $\lambda_{-}(s)$ has a minimum at

$$
s_{m}=\sqrt{\frac{C^{2}}{4 D^{2}}-\frac{\gamma^{2}}{C^{2}}} .
$$

At this value of $s, \lambda_{-}(s)$ is equal to

$$
\lambda_{m}=3 \gamma-\frac{C^{2}}{4 D}-\frac{\gamma^{2} D}{C^{2}} .
$$


Using relations (B6), we find that the minimum value of $\lambda_{-}(s)$ is equal to

$$
\lambda_{m}=\frac{7}{16} D \eta^{2}
$$

and it occurs at

$$
s_{m}=\frac{\sqrt{15}}{4} \eta
$$

Fig. 7 shows $\lambda_{ \pm}(s)$ given by Eq. (C10) as a function of $s$.

In order to obtain the general solution of Eqs. (C1. C2), we should integrate the special solutions (C3 C4) over positive parameter $s$ taking into account relations (C7,C9). Two arbitrary functions $C_{ \pm}(s)$ entering Eqs. (C7 4 C9 can be found from specified initial conditions for the radial and $z$-components of polarization in the form of its Fourier-Bessel transforms

$$
\begin{aligned}
& \tilde{S}_{r}(s)=\int_{0}^{+\infty} d r r S_{r}(r, 0) J_{1}(s r), \\
& \tilde{S}_{z}(s)=\int_{0}^{+\infty} \operatorname{drr} S_{z}(r, 0) J_{0}(s r) .
\end{aligned}
$$

As a result of algebraic transformations, we obtain the solution of the initial value problem (C1) C2 in the case of the infinite plane

$$
\begin{aligned}
S_{r}(r, t) & =\int_{0}^{+\infty} d s s G_{r r}(r, t, s) \tilde{S}_{r}(s) \\
& +\int_{0}^{+\infty} d s s G_{r z}(r, t, s) \tilde{S}_{z}(s), \\
S_{z}(r, t) & =\int_{0}^{+\infty} d s s G_{z r}(r, t, s) \tilde{S}_{r}(s) \\
& +\int_{0}^{+\infty} d s s G_{z z}(r, t, s) \tilde{S}_{z}(s),
\end{aligned}
$$

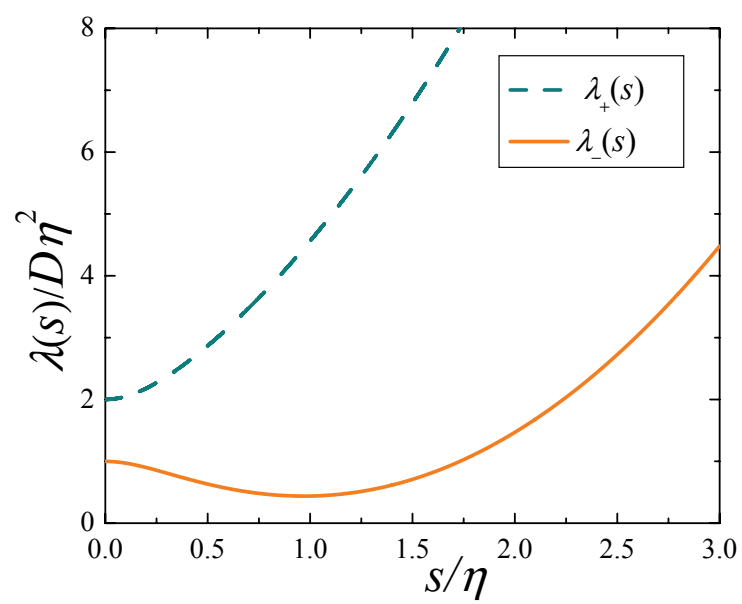

FIG. 7. (Color online) $s$-dependence of inverse relaxation times $\lambda_{ \pm}(s)$. The minimum of $\lambda_{-}(s)$ corresponds to the wave vector giving the longest relaxation time. where the Green functions $G_{r r}, G_{z r}, G_{r z}, G_{z z}$ are defined as follows

$$
\begin{array}{r}
G_{r r}(r, t, s)=J_{1}(r s)\left[\cosh \left(t \sqrt{\gamma^{2}+C^{2} s^{2}}\right)\right. \\
+\gamma \frac{\left.\sinh \left(t \sqrt{\gamma^{2}+C^{2} s^{2}}\right)\right]}{\left.\sqrt{\gamma^{2}+C^{2} s^{2}}\right]} e^{-\left(D s^{2}+3 \gamma\right) t}, \\
G_{r z}(r, t, s)=-C J_{1}(r s) \frac{\sinh \left(t \sqrt{\gamma^{2}+C^{2} s^{2}}\right)}{\sqrt{\gamma^{2}+C^{2} s^{2}}} \\
G_{z r}(r, t, s)=-C J_{0}(r s) \frac{\sinh \left(t \sqrt{\gamma^{2}+C^{2} s^{2}}\right)}{\sqrt{\gamma^{2}+C^{2} s^{2}}} \\
\times s e^{-\left(D s^{2}+3 \gamma\right) t}, \\
G_{z z}(r, t, s)=J_{0}(r s)\left[\cosh \left(t \sqrt{\gamma^{2}+C^{2} s^{2}}\right)\right. \\
\sinh \left(t \sqrt{\gamma^{2}+C^{2} s^{2}}\right) \\
\left.\sqrt{\gamma^{2}+C^{2} s^{2}}\right]
\end{array}
$$

Substituting the initial conditions for the radial spin helix (1]3) into Eqs. (C15C16) and performing integration we find that the generalized functions $\tilde{S}_{r}(s)$ and $\tilde{S}_{z}(s)$, which correspond to the initial conditions (11) and (3), act as follows

$$
\begin{gathered}
\int_{0}^{+\infty} d s s F(s) \tilde{S}_{r}(s)=-S_{0} \frac{d}{d k} \int_{0}^{k} d s \frac{k F(s)}{\sqrt{k^{2}-s^{2}}}, \\
\int_{0}^{+\infty} d s s F(s) \tilde{S}_{z}(s)=S_{0} \frac{d}{d k} \int_{0}^{k} d s \frac{s F(s)}{\sqrt{k^{2}-s^{2}}},
\end{gathered}
$$

where $F(s)$ is a smooth enough function.

Substituting Eqs. C23C24 into the general solution (C17C18) and taking into account the expressions for the Green functions (C19,C22), we obtain the explicit formulae for the solution of the drift-diffusion equations (C1,C2) for the initial conditions (1, 3]):

$$
\begin{gathered}
S_{r}(r, t)=-S_{0} \frac{d}{d k} \int_{0}^{k} \frac{d s J_{1}(s r)}{\sqrt{k^{2}-s^{2}}}\left[k \cosh \left(t \sqrt{\gamma^{2}+C^{2} s^{2}}\right)\right. \\
\left.+\left(k \gamma+C s^{2}\right) \frac{\sinh \left(t \sqrt{\gamma^{2}+C^{2} s^{2}}\right)}{\sqrt{\gamma^{2}+C^{2} s^{2}}}\right] e^{-\left(D s^{2}+3 \gamma\right) t},(\mathrm{C} 25) \\
S_{z}(r, t)=S_{0} \frac{d}{d k} \int_{0}^{k} \frac{d s s J_{0}(s r)}{\sqrt{k^{2}-s^{2}}}\left[\cosh \left(t \sqrt{\gamma^{2}+C^{2} s^{2}}\right)\right. \\
\left.+(k C-\gamma) \frac{\sinh \left(t \sqrt{\gamma^{2}+C^{2} s^{2}}\right)}{\sqrt{\gamma^{2}+C^{2} s^{2}}}\right] e^{-\left(D s^{2}+3 \gamma\right) t} . \quad(\mathrm{C} 26)
\end{gathered}
$$

\section{Appendix D: Relaxation of plain spin helix}

In the case of the plane spin helix defined by the initial conditions (4,6), the solution of the drift-diffusion 
equations (B3) has the same harmonical spatial dependence at any moment of time. Therefore, we can seek the solution of this system of equations in the form

$$
\begin{array}{r}
S_{x}(x, t)=A(k, t) \sin (k x), S_{y}(x, t)=0, \\
S_{z}(x, t)=B(k, t) \cos (k x) .
\end{array}
$$

Substituting expressions (D1,D2) into the system (B3) B5) we obtain the same system of ordinary differential equations (C5) C6) for the functions $A(k, t)$ and $B(k, t)$ as in the axially symmetric case. Eqs. (D1,D2) together with Eqs. (C7) C9 determine solutions of the drift-diffusion equations (B3) B5) with such a specific harmonic spatial dependence. The arbitrary amplitudes $C_{+}$

* pershin@physics.sc.edu

1 D. D. Awschalom, N. Samarth, and D. Loss, eds., Semiconductor Spintronics and Quantum Computation (SpringerVerlag, 2002).

2 I. Zutic, J. Fabian, and S. Das Sarma, Rev. Mod. Phys. 76, 323 (2004).

3 M. I. Dyakonov and V. I. Perel', Sov. Phys. Solid State 13, 3023 (1972).

${ }^{4}$ M. I. Dyakonov and V. Y. Kachorovskii, Sov. Phys. Semicond. 20, 110 (1986).

5 A. A. Kiselev and K. W. Kim, Phys. Rev. B 61, 13115 (2000).

6 E. Y. Sherman, Appl. Phys. lett 82, 209 (2003).

7 M. Q. Weng, M. W. Wu, and Q. W. Shi, Phys. Rev. B 69, 125310 (2004).

8 Y. V. Pershin and V. Privman, Phys. Rev. B 69, 073310 (2004).

9 Y. V. Pershin, Phys. Rev. B 71, 155317 (2005).

10 L. Jiang, M. Weng, M. Wu, and J. Cheng, J. Appl. Phys. 98, 113702 (2005).

11 B. A. Bernevig, J. Orenstein, and S.-C. Zhang, Phys. Rev. Lett. 97, 236601 (2006).

12 P. Schwab, M. Dzierzawa, C. Gorini, and R. Raimondi, and $C_{-}$are calculated from the initial conditions (Eqs. (4460)). Finally, the solution of drift-diffusion equations (B3) B5) for the plane spin helix is given by

$$
\begin{array}{r}
S_{x}(x, t)=-S_{0} \sin (k x)\left[\cosh \left(t \sqrt{\gamma^{2}+C^{2} k^{2}}\right)\right. \\
\left.+(C k+\gamma) \frac{\sinh \left(t \sqrt{\gamma^{2}+C^{2} k^{2}}\right)}{\sqrt{\gamma^{2}+C^{2} k^{2}}}\right] e^{-\left(D k^{2}+3 \gamma\right) t}, \\
S_{z}(x, t)=S_{0} \cos (k x)\left[\cosh \left(t \sqrt{\gamma^{2}+C^{2} k^{2}}\right)\right. \\
\left.+(C k-\gamma) \frac{\sinh \left(t \sqrt{\gamma^{2}+C^{2} k^{2}}\right)}{\sqrt{\gamma^{2}+C^{2} k^{2}}}\right] e^{-\left(D k^{2}+3 \gamma\right) t} .
\end{array}
$$

Phys. Rev. B 74, 155316 (2006).

13 J. D. Koralek, C. P. Weber, J. Orenstein, B. A. Bernevig, S.-C. Zhang, S. Mack, and D. D. Awschalom, Nature 458, 610 (2009).

14 P. Kleinert and V. V. Bryksin, Phys. Rev. B 79, 045317 (2009).

15 M. Duckheim, D. L. Maslov, and D. Loss, Phys. Rev. B 80, 235327 (2009).

16 I. V. Tokatly and E. Y. Sherman, Ann. Phys. 325, 1104 (2010).

17 Y. Bychkov and E. Rashba, JETP Lett. 39, 78 (1984).

18 G. Dresselhaus, Phys. Rev. 100, 580 (1955).

19 C. P. Weber, J. Orenstein, B. A. Bernevig, S.-C. Zhang, J. Stephens, and D. D. Awschalom, Phys. Rev. Lett. 98, 076604 (2007).

20 S. Saikin, Y. Pershin, and V. Privman, IEE-Proc. Circ. Dev. Syst. 152, 366 (2005).

21 A. R. Cameron, P. Riblet, and A. Miller, Phys. Rev. Lett. 76, 4793 (1996).

${ }^{22}$ F. Reif, Fundamentals of Statistical and Thermal Physics (McGraw-Hill, 1965).

23 Y. Pershin, Phys. E 23, 226 (2004). 